\title{
COHERENT ENSEMBLE AVERAGING TECHNIQUES FOR IMPEDANCE CARDIOGRAPHY
}

\author{
BARRY E. HURWITZ, LIANG-YU SHYU, SRIDHAR P. REDDY, \\ NEIL SCHNEIDERMAN AND JOACHIM H. NAGEL
}

\author{
Behavioral Medicine Research Center, Departments of Psychology \& Biomedical \\ Engineering, University of Miami, Coral Gables, Florida 33124
}

\section{ABSTRACT}

\begin{abstract}
EKG synchronized ensemble averaging of the impedance cardiogram tends to blur or suppress signal events due to signal jitter or event latency variability. Although ensemble averaging provides some improvement in the stability of the signal and signal to noise ratio under conditions of nonperiodic influences of respiration and motion, coherent averaging techniques were developed to determine whether further enhancement of the impedance cardiogram could be obtained. Physiological signals were obtained from sixteen male and female subjects during resting conditions, while delivering a speech and while undergoing submaximal bicycle exercise. Results indicated that improved resolution of $\mathrm{dZ} / \mathrm{dt}$ signal events could be obtained using coherent ensemble averaging. Although some improvement in precision of event location was obtained, most enhancement of the impedance cardiogram occurred in measurement of the amplitude of the $\mathrm{dZ} / \mathrm{dt}$ maximum (ejection velocity) during speaking and exercise conditions. Validated increases in $\mathrm{dZ}$ dt maximum exceeding 20\% were obtained in some subjects with coherent averaging, suggesting that the diagnostic utility of impedance cardiography can be improved by using this technique.
\end{abstract}

(Supported by NHLBI research grants, HLA1335 and HL36588.)

\section{INTRODUCTION}

Validation studies have shown that impedance cardiography (ICG) can provide a reasonably accurate estimate of stroke volume (SV) [see review 1]. The ICG, however, is susceptible to artifactual influences of respiration and motion. These influences have been countered by ensemble averaging of the ICG, which when performed across several respiratory cycles effectively filters signal noise, and respiratory and motion artifact and provides an adequate estimate of SV, cardiac output and cardiac time intervals $[4,5,6]$. Moreover, measures derived from ensemble averaged waveforms do not statistically differ from averages of beat-by-beat measures over the identical time period [7].

Ensemble averaging is a technique in which the ICG is averaged on a consecutive beatby-beat basis and temporally synchronized with the R-wave of the electrocardiogram (EKG) (see Figure 1). This technique is supposed to provide a coherent average improving signal to noise ratio. However, ensemble averaging suffers from the difficulties inherent with intrasubject variability of signal shape and event latency. Events in the ICG signal (i.e., the B point which reflects aortic opening, the dZ/dtmax which reflects the peak systolic ejection rate and the $X$ wave which reflects aortic closure and end of ventricular ejection) can be blurred or even disappear in the ensemble averaged signal. For example, $\mathrm{B}$ and $\mathrm{X}$ waves tend to flatten making them less distinct and a broadening of the $\mathrm{dZ} / \mathrm{dt}$ peak can occur. Therefore, ensemble averaging does not actually provide coherent averaging unless there are absolutely stable signal event time intervals. The purpose of this paper was to compare another signal processing technique, coherent ensemble averaging, with ensemble averaging of the ICG under conditions of rest and conditions of cardiorespiratory activation elicited by public speaking or bicycle exercise. Coherent averaging differs from ensemble averaging in that the distinct components of the impedance waveform are aligned, averaged, and then as with ensemble averaging adjusted 
relative to the $\mathrm{R}$ wave of the EKG. However, the event adjustment is based upon the average temporal location derived from beat-by-beat detection of the three signal events: $B$ point, dZ/dtmax and the minimum of the $X$ wave (see Figure 1). Therefore, by aligning and averaging the events, coherent averaging should provide a more enhanced and precise representation of the waveform components than ensemble averaging, by producing an averaged waveform that retains the advantage of enhanced signal to noise ratio and is relatively free from beat-to-beat variation in event latency.

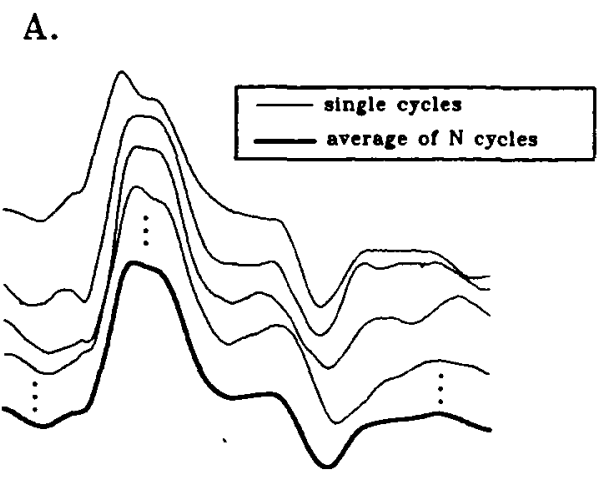

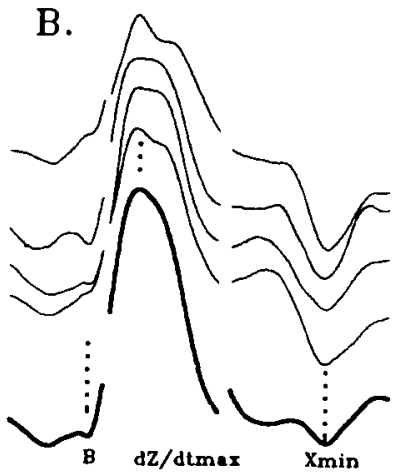

Figure 1. Panel A: R-wave triggered ensemble average. Panel B: coherent averagingevent alignment (B, dZ/dtmax and $\mathrm{Xmin}$ ), averaging, adjustment relative to $\mathrm{R}$-wave.

\section{METHOD}

\section{Subjects}

Sixteen ( 8 men and 8 women) Caucasian native born, U.S. citizens, with mean ( \pm SD) age of $44.6 \pm 7$ yrs (range 26-53 yrs) served as subjects. Prior to the testing session, after informed consent, a medical history was taken with all subjects reporting no cardiopulmonary or other medical disorders. This was confirmed by physical examination, fasting blood chemistry analysis and twelve lead EKG. Mean \pm SD height and weight were respectively, $162.3 \pm 4 \mathrm{~cm}$ and $73.5 \pm 21 \mathrm{~kg}$ for females and $174.2 \pm 5 \mathrm{~cm}$ and 83.0 $\pm 9 \mathrm{~kg}$ for males. All subjects had a diastolic blood pressure (BP) less than $90 \mathrm{~mm} \mathrm{Hg}$.

\section{Procedure}

The physiological data used in this study were collected between 0900 and 1400 on the testing day. A heparinized butterfly blood withdrawal needle (Kowarski-Cormed thromboresistant blood withdrawal needle (18 ga) and tubing set) was inserted into an antecubital vein and attached to a small $(10 \times 9 \mathrm{~cm}$ ) portable peristaltic pump (Cormed ML6). During the testing session BP, EKG, phonocardiogram (PCG), respiration, the derivative of the pulsatile impedance signal (dZ/dt) and mean thoracic impedance (Zo) were recorded. Blood was collected for hematocrit and hence blood resistivity determination while subjects were at rest or engaged in the behavioral challenges. Physiological measurements were obtained during three periods: resting baseline, evaluative speech 
stressor and submaximal bicycle exercise. Data were collected during the last 6-min of a 20-min period of rest while seated in a chair, during a 3-min period in which the subjects were asked to deliver into a video recording camera a speech on a threatening topic, which they were told would be subsequently evaluated [8], and during a 9-min seated-bicycle exercise in which the workload of the bicycle was computer-adjusted to maintain the subjects' heart rate at $60 \%$ of maximum level [9]. These measurement periods were presented to the subjects in this fixed order. Three 30-s samples of physiological data evenly spaced throughout each of these periods were selected for comparison of the two waveform averaging computer applications.

\section{Physiological Recording Apparatus and Signal Transduction}

Measurements of BP were obtained using a Critikon Dinamap Adult/Pediatric Vital Signs Monitor. The BP measures will not be reported here. EKG, PCG, ICG and respiration were recorded continuously with a Grass polygraph. Either a standard EKG Lead I or II configuration was selected. The ICG was derived using a tetrapolar aluminum and mylar tape electrode configuration. Lead 2 was placed just superior to the suprasternal notch of the thorax, at the base of the neck. Lead 1 was placed precisely $3 \mathrm{~cm}$ superior to lead 2 on the upper neck. Lead 3 was placed around the thorax overlaying the xiphosternal joint. Lead 4 was placed on the lower thorax precisely $5 \mathrm{~cm}$ inferior to lead 3 . These four ICG leads were placed around the body, while the subject was standing erect, such that the impedance leads $1 \& 2$ were parallel to each other and leads $3 \& 4$ were parallel to the floor. This was done to ensure uniform current spread over the body. An alternating current of $4 \mathrm{~mA}$ at $100 \mathrm{kHz}$ was passed through the outer electrodes and the signals were recorded from the inner two electrodes. The two signals derived from the ICG signal were Zo and dZ/dt. The PCG was recorded to determine heart sounds by placing a phonotransducer (Hewlett Packard-21050A) on the cardiac window in the second or fourth intracostal space just to the left of the sternum. Respiration was recorded by placing a mercury strain-gauge on the abdomen below the fourth impedance lead.

The EKG, PCG, and respiration signal were relayed from the polygraph to an $A / D$ converter (DT 2801) at $1 \mathrm{kHz}$ sampling rate and stored in the IBM PC-AT computer. The $\mathrm{dZ} / \mathrm{dt}$ signal was also sampled at $1 \mathrm{kHz}$ and relayed directly from the Minnesota Impedance Cardiograph to the $A / D$ converter so that the impedance signal would be free of the attenuating effects imposed by polygraphic high frequency filtering. An impedance calibration signal was also stored in the computer for later conversion of the $\mathrm{dZ} / \mathrm{dt}$ signal to $\mathrm{Ohms} / \mathrm{s}$. The EKG, ICG, and PCG were collected continuously during baseline periods and task periods, and stored as 30-s samples on the computer. Blood was collected as integrated 3-min samples during the baseline periods and tasks.

\section{Coherent and Ensemble Averaging Procedures}

The computer program calculated the ensemble average and coherent average during a single pass of a 30-s sample of EKG and dZ/dt. Ensemble averaging initially required the detection of the R-wave of the EKG. The dZ/dt samples for each cycle starting from $R$ wave detection, were summed into a storage buffer. After the information from the last cycle was stored the sum was divided by the total number of cycles in the sample and this ensemble average was presented to the graphics terminal display and stored for later analysis. The coherent average was similarly developed for each $30-\mathrm{s}$ sample. However, the coherent averaging process differed from the ensemble averaging method as follows. a) the signal events were detected on a beat-by-beat basis which required additional signal processing techniques; and $b$ ) these signal events synchronized the waveform averaging. Specifically, before coherent averaging, a template was generated by ensemble averaging the first 10 cycles of the $\mathrm{dZ} / \mathrm{dt}$ waveform. The signal events, B point, $\mathrm{dZ} / \mathrm{dtmax}$ and $\mathrm{X}$ wave minimum were detected on this ensemble average and these events were windowed 
( $\pm 50 \mathrm{~ms}$ ) to create a template of each event. Then, consequent to the EKG R-wave detection for each cycle, a matched filtering technique was employed, which calculated the cross correlation between the template and the incoming $\mathrm{dZ} / \mathrm{dt}$ waveform to detect the signal events. The precision of these signal detection techniques have been previously described [10]. The template was updated upon each detection enhancing detection reliability, especially in cases of slowly changing waveform topography. To ensure complete accuracy of event detection the program permitted the operator to correct interactively any events scored incorrectly. Once each of the three events had been detected the time point relative to the $R$ wave was stored for later adjustment following the averaging of the events, which were summed into a storage buffer and divided by the total number of cycles. The coherent averages of each of the three events were then temporally adjusted relative to the mean interval from the $R$ wave, displayed to the terminal screen and stored for later analysis.

The temporal difference between the beat-by-beat onset of each signal event relative to the onset of the event in the signal template was calculated to provide an index of the variation of the signal events. In addition, several indices of myocardial performance were derived from the computer samples of EKG and ICG from both the ensemble and coherent averages for later statistical comparison. Left ventricular ejection time (LVET) was derived by measuring the interval (ms) between the B-wave local minimum and the onset of the $X$ wave of the $\mathrm{dZ} / \mathrm{dt}$. The second heart sound from the PCG, which reflects aortic valve closure, was used only to aid in verifying that the $\mathrm{dZ} / \mathrm{dt} \mathrm{X}$-wave was properly identified. The ejection velocity (dZ/dtmax) is measured in Ohms/s and was calculated as the difference in amplitude between the peak of the dZ/dt signal and the zeroline divided by the amplitude of the $\mathrm{dZ} / \mathrm{dt}$ calibration signal. SV was derived using the Kubicek formula [11] as follows: $\mathrm{SV}=$ tho $*(\mathrm{~L} / \mathrm{Zo})^{2} * \mathrm{LVET} * \mathrm{dZ} / \mathrm{dmax}$. Rho is the blood resistivity in Ohms.cm and was calculated using the formula: tho $=53.2 \mathrm{e}^{(0.022) \mathrm{HCT}}$, where HCT is the hematocrit as measured from the collected blood samples. The closest HCT in time with the 30-s period was used to calculate rho. The mean Zo from each 30 -s sample was calculated. The electrode distance $(\mathrm{L})$ in $\mathrm{cm}$ between lead 2 and lead 3 was measured while the subject was seated prior to the first baseline and bicycle exercising periods.

\section{RESULTS}

The data analysis (repeated measures analysis of variance) consisted of initially determining whether each of the three periods of measurement differed in the degree of variation of the signal events on a beat-by-beat basis. Then the coherent and ensemble averaging techniques were compared during the baseline, speech and exercise periods to determine on the averaged waveforms whether differences were produced in: a) event location; and b) LVET, dZ/dimax or SV.

The beat-to-beat temporal location of the detected signal events was determined relative to the temporal point of the event within the original template established in the matched filtering procedure. Table 1 presents the mean $( \pm S D)$ variation in the location of these signal events detected during the beat-by-beat coherent averaging process. As expected, the variation in the events during the resting baseline period was significantly less than during the speech and exercise periods ( $p<.001$ ). Moreover, significantly greater variation was observed during the speech than the exercise task in B point $(p<.001)$ and $X$ min $(p<.001)$, with no difference between these tasks in influencing the $\mathrm{dZ} / \mathrm{dt}$ peak location. Therefore, most improvement in the signal event waveforms when using coherent averaging would be expected during the speaking and exercise periods, since these are the periods of greatest event variation. 
Table 1. Mean ( \pm SD) Variation in Signal Event Location (ms) Relative to the Initial Template Event Location.

$\begin{array}{llll}\text { Event } & \text { Resting Baseline Speech } & \text { Bicycle Exercise }\end{array}$

$\begin{array}{lrrr}\mathrm{B} & 3.5 \pm 2 & 6.8 \pm 3 & 5.1 \pm 3 \\ \mathrm{dZ} / \mathrm{dtmax} & 4.7 \pm 3 & 10.5 \pm 6 & 8.6 \pm 5 \\ \mathrm{X} \min & 7.1 \pm 4 & 14.2 \pm 6 & 10.3 \pm 4\end{array}$

Table 2 depicts the mean \pm SD difference and absolute value of the difference between the coherent and ensemble averaging methods in the location of the signal events on the averaged waveform. Although there was no significant difference between methods in the location of the peak of the $\mathrm{dZ} / \mathrm{dt}$, there were significant differences between the methods for B point ( $p<.005)$ and $X_{\min }(p<.05)$ locations. Indeed, systematic differences were observed with the coherent average method producing a $B$ point that was about $1.4 \mathrm{~ms}$ later than the ensemble average. Whereas, the Xmin was located approximately 6-7 ms earlier in the coherent waveform than in the ensemble average. There were only small differences

Table 2. Mean ( \pm SD) Difference and Absolute Difference Between Coherent and Ensemble Averaging Techniques in Signal Event Location (ms).

\begin{tabular}{lcccccc}
\hline Event & \multicolumn{2}{c}{$\begin{array}{c}\text { Resting Baseline } \\
\text { Diff }\end{array}$} & $\begin{array}{c}\text { Speech } \\
{[\text { Diff] }}\end{array}$ & Diff & [Diff & \multicolumn{2}{c}{ Bicycle Exercise } \\
& & & & & & \\
& & & & & & \\
\hline & $1.6 \pm 3$ & $2.7 \pm 2$ & $2.3 \pm 5$ & $3.9 \pm 4$ & $3.2 \pm 5$ & $3.7 \pm 4$ \\
B & $0.1 \pm 3$ & $1.4 \pm 2$ & $2.0 \pm 7$ & $4.7 \pm 6$ & $-0.2 \pm 6$ & $3.8 \pm 4$ \\
XZ/dtmax & $-6.1 \pm 15$ & $9.4 \pm 13$ & $-6.8 \pm 13$ & $10.3 \pm 10$ & $-1.0 \pm 8$ & $5.4 \pm 6$ \\
\hline
\end{tabular}
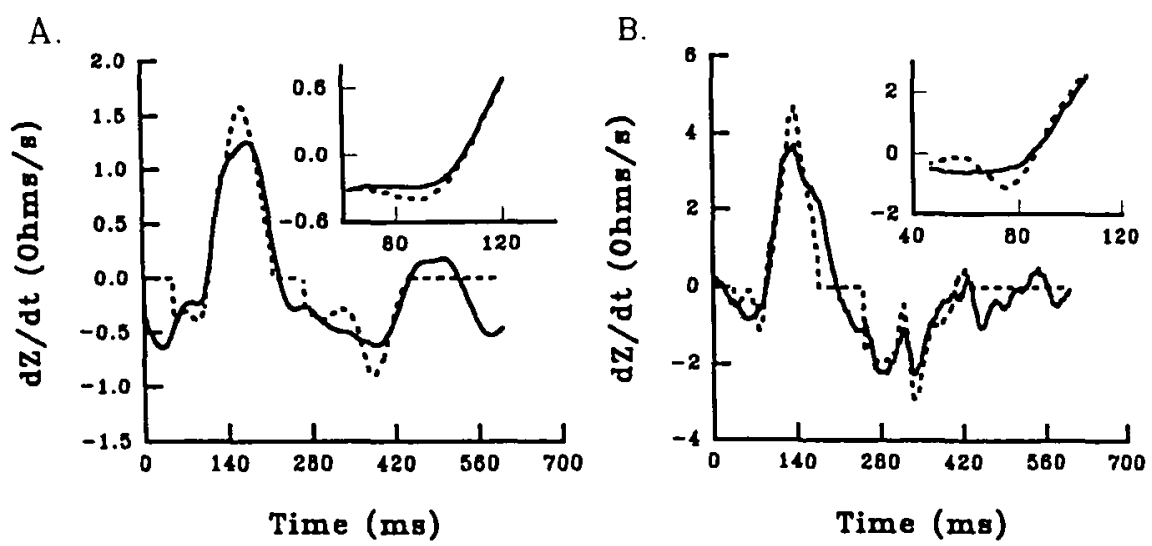

Figure 2. Two cases ( $\# 43 \&$ \& \#65) of enhancement of $B$ point during speech (A) and exercise (B) using coherent averaging (dotted) compared with ensemble averaging (solid). 

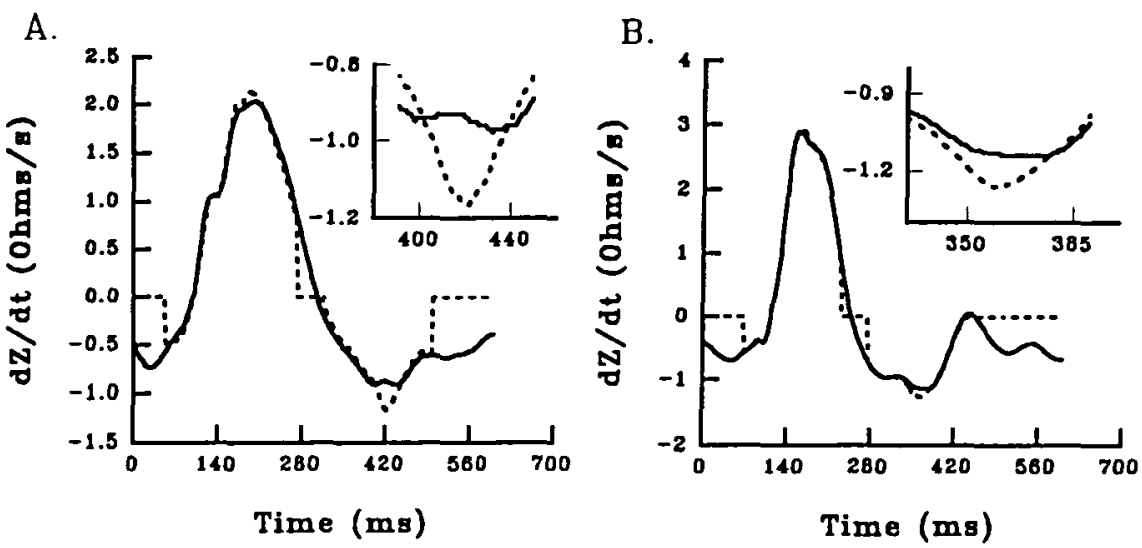

Figure 3. Two cases (\#47 \& \#77) of enhancement of $X$ minimum during speech $(A)$ and exercise (B) using coherent averaging (dotted) compared with ensemble averaging (solid).

obtained between methods during the exercise period in the location of the Xmin. Although the averaging methods, in general, produced small temporal differences in the event locations, inspection of the averaged waveforms (see examples in Figures 2 and 3) reveals that the coherent averaging technique enhanced the resolution of the waveform events.

Since the B point occurred later and the $X_{\min }$ earlier in the coherent average than the ensemble average waveforms, the LVET values should be shorter following application of coherent averaging. As can be seen in Table 3 coherent averaging did produce significantly shorter LVET than the ensemble averaging method $(p<.005)$, by approximately $7 \mathrm{~ms}$. This difference was unaffected by period of measurement. Since LVET is directly related to SV in the Kubicek equation, the shorter LVET obtained using coherent averaging would have the effect of reducing $S V$ by about $2-3 \%$.

Table 3. Comparison of Mean ( \pm SD) \& Percentage Difference of LVET (ms), dZ/dmax $(\mathrm{Ohms} / \mathrm{s})$ and SV (ml) using Coherent $(\mathrm{COH})$ and Ensemble (ENS) Averaging Methods.

Measure Resting Baseline Speech Bicycle Exercise

$\mathrm{COH}$ ENS \%Diff $\mathrm{COH}$ ENS \%Diff $\mathrm{COH}$ ENS \%Diff

$\begin{array}{lrrrrrrrrr}\text { LVET } & 309.4 \pm 31 & 317.1 \pm 42 & -2.4 & 292.6 \pm 44 & 301.7 \pm 50 & -3.0 & 261.7 \pm 25 & 265.9 \pm 26 & -1.6 \\ \text { dZddtmax } & 2.04 \pm 1 & 2.01 \pm 1 & 1.3 & 2.25 \pm 1 & 2.14 \pm 1 & 5.1 & 2.83 \pm 1 & 2.65 \pm 1 & 6.8 \\ \text { SV } & 59.9 \pm 43 & 60.3 \pm 43 & -0.7 & 63.8 \pm 37 & 62.1 \pm 37 & 2.7 & 70.7 \pm 37 & 67.9 \pm 36 & 4.1\end{array}$

Although many laboratories measure the ejection velocity as the amplitude of $\mathrm{dZ} / \mathrm{dt}$ relative to the $B$ point, the ejection velocity reported in this study was measured from the peak of the dZ/dt relative to the zero line in order to determine the effect of the signal processing methods unconfounded by differences in the location of the B point. It was in this measure that the most prominent effect of the coherent averaging technique could be discriminated, especially in individual cases that exhibit more temporal variability in dZ/dtmax (see Figure 4). Table 3 displays the mean dZ/domax values during the periods of measurement contrasting the averaging methods. While there was no difference in 
$\mathrm{dZ} / \mathrm{dtmax}$ between methods under the stable conditions of the resting baseline, significant differences were found during speech $(p<.001)$ and exercise tasks $(p<.05)$. coherent averaging during these two conditions of relatively greater respiratory and motoric instability produced a $5-7 \%$ increase in $\mathrm{dZ} / \mathrm{dtmax}$ compared with ensemble averaging. Figure 4 shows that the effect of coherent averaging in enhancing the dZ/dt peak in some subjects while speaking or exercising can be quite substantial, exceeding $20 \%$. The combined coherent averaging effect on SV of the shortened LVET and the increased dZdtmax is shown in Table 3. Although these effects have opposite influences on SV, the decreases in LVET were not sufficient to counter the increases in dZ/dtmax, with significant elevations in SV of $3-4 \%$ maintained during speech $(p<.01)$ and exercise periods $(p<.05)$.

A.

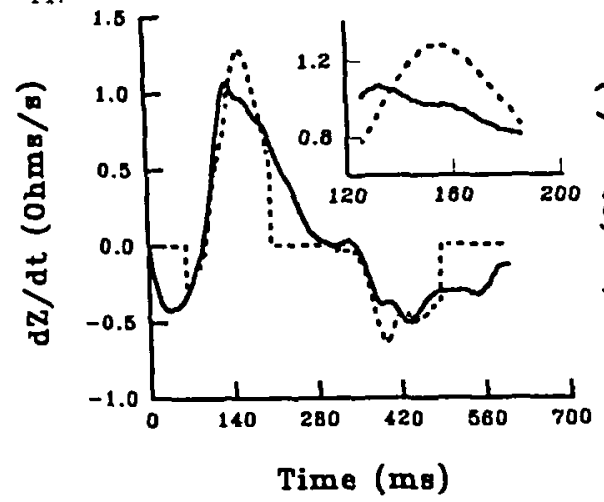

B.

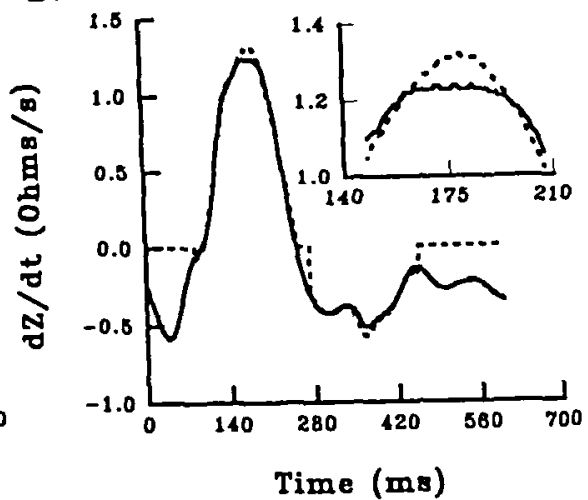

Figure 4. Two cases (\#66 \& \#43) of enhancement of dZ/dmax during speech (A) and exercise (B) using coherent averaging (dotted) compared with ensemble averaging (solid).

\section{DISCUSSION}

We have shown that the beat-to-beat variations of the B point, $d Z / d$ max and $X \min$ over a 30-s sampling interval are quite stable when the subject is at rest. The respiratory and movement influences inherent in the speech and exercise challenges, however, produced more variation in the signal events, mostly the Xmin and dZJdt peak. Systematic changes in the averaged waveform occurred upon application of coherent averaging with slightly later detection of the $B$ point and earlier detection of the $X$ min relative to the $R$ wave of the EKG. These combined effects resulted in a reduction in LVET of about $7 \mathrm{~ms}$ due to coherent averaging. Although the effect of coherent averaging on the temporal location of the signal events was not very substantial, inspection of Figures 2-4 reveals the clear enhancement of the resolution of each of the signal events. Therefore, while the precision of the detection of the events is only marginally improved, the reliability of detection of the events, especially under circumstances of unusual respiratory and motion modulation would appear to be improved. The greatest effect of coherent averaging on the impedance cardiogram was to increase the dZ/dtmax, which on average increased $5-7 \%$, although in some subjects improvements exceeded $20 \%$. Figure 4 clearly shows how the $\mathrm{dZ} / \mathrm{dt}$ peak can be broadened after ensemble averaging. Coherent averaging removes this effect, enhancing the resolution of the $\mathrm{dZ} / \mathrm{dt}$ peak. On the basis of the improvements in the dZ/dtmax alone it would appear that the use of coherent averaging techniques could improve the reliability and validity of SV measurement, especially under nonsteady state conditions. 
Comparisons of impedance cardiography with more well established invasive techniques have shown high correlations when averaged across a group of individuals [1]. However, when applied in individual cases the correlations have been much poorer [12, 13]. It is quite clear from the presentation of the individual cases in this study that coherent averaging may substantially alter SV. Therefore, differences between ensemble and coherent averaging techniques would tend to be minimized when data sets are averaged over many subjects. However, on a single case basis or when improved reliability is needed for diagnostic purposes improved measurement may be obtained by using coherent averaging. Moreover, with the enhancement of the signal-to-noise ratio on each of the relevant events, the test-retest reliability may be improved, especially if there are differences in noise level when retesting. In conclusion, coherent averaging as a signal processing technique retains the advantages of ensemble averaging including event enhancement and nonperiodic waveform attenuation (e.g., respiratory, motion, and electrical noise artifact). Coherent averaging also provides the advantage of reducing the effect of variation in event latency, thereby enhancing individual landmarks in the ICG.

\section{REFERENCES}

[1] A. Sherwood et al., "Committee report: Methodological guidelines for impedance cardiography," Psychophysiology, in press.

[2] E. Enghoff and O. Lovheim, "A comparison between the transthoracic electrical impedance method and the direct Fick and dye dilution methods for cardiac output measurements in man," Scand. J. Clin. Lab. Invest. vol. 39, p. 585-590, 1979.

[3] W.V. Judy et al., "Comparative evaluation of the thoracic impedance and isotope dilution methods for measuring cardiac output," Aerosp. Med. vol. 40, p. 532-536, 1969.

[4] M. Muizi et al., "Determination of cardiac output using ensemble-averaged impedance cardiograms," J. Appl. Physiol. vol. 58, p. 200-205, 1985.

[5] Y. Miyamoto et al., "Continuous determination of cardiac output during exercise by the use of impedance plethysmography," Med. Biol. Eng. Comput. vol. 19, p. 638-644, 1981.

[6] D.S. Sheps et al., "Continuous noninvasive monitoring of left ventricular function during exercise by thoracic impedance cardiography-automated derivation of systolic time intervals," Amer. Heart J. vol. 103, p. 519-524.

[7] R.M. Kelsey and W. Guethlein, "An evaluation of the ensemble averaged impedance cardiogram." Psychophysiology, in press.

[8] P.G. Saab et al., "Premenopausal and postmenopausal women differ in their cardiovascular and neuroendocrine responses to behavioral stressors," Psychophysiology, vol. 26, p. 270-280, 1989.

[9] D.H. VanDercar et al., "Microcomputer automated system for measuring systolic time intervals in response to exercise and a psychophysiological task," Psychophysiology, vol. 26, p. 703-711, 1988.

[10] J.H. Nagel et al, "New signal processing techniques for improved precision of noninvasive impedance cardiography," Ann. Biomed. Eng. vol. 17, p. 517-534, 1989.

[11] W.G. Kubicek et al., "Development and evaluation of an impedance cardiac output system. Aerosp. Med. vol. 39, p. 248-252, 1966.

[12] P.L. Appel et al., "Comparison of measurements of cardiac output by bioimpedance and thermodilution in severely ill surgical patients," Crit. Care Med. vol. 14, p. 933-935, 1986.

[13] D.P. Bernstein, "Continuous non-invasive real-time monitoring of stroke volume and cardiac output by thoracic electrical bioimpedance," Crit. Care Med. vol. 14, 898-901, 1986. 